\title{
Atopic dermatitis therapy breakthrough on the horizon?
}

Phase I and II studies published recently suggest that dupilumab, a monoclonal antibody that targets a subunit of the interleukin-4 (IL-4) and IL-13 receptors, has the potential to become the first systemic therapy for atopic dermatitis to be approved by the US Food and Drug Administration (FDA) (N. Engl.J. Med. 371, 130-139; 2014). Dupilumab, which is being developed by Regeneron and Sanofi, has also previously shown efficacy in a Phase II trial for asthma (N. Engl.J. Med. 368, 2455-2466; 2013).

Atopic dermatitis, a T helper $2\left(\mathrm{~T}_{\mathrm{H}} 2\right)$-mediated inflammatory skin condition, affects an estimated $10-20 \%$ of children and $3-10 \%$ of adults in the United States alone. Of those, $20 \%$ have moderate-to-severe forms of the disease; patients enrolled in the dupilumab trials - three monotherapy trials and one trial of dupilumab plus topical glucocorticoids — fell into this category and had itchy dermatitis covering $~ 40-50 \%$ of their bodies.

"The efficacy of dupilumab was remarkable," says Lisa Beck, Professor at the Department of Dermatology, University of Rochester Medical Center, and the lead author of the study. For example, in the 12-week monotherapy trial, significantly more patients receiving dupilumab than those receiving placebo had $a \geq 50 \%$ reduction in their Eczema Area and Severity Index (EASI) score (85\% versus 35\%, respectively), clearing of skin lesions ( $40 \%$ versus $7 \%$ ), and decreased itching ( $55.7 \%$ versus $15.1 \%$ ). All of the patients treated with dupilumab plus glucocorticoids had a $\geq 50 \%$ reduction in EASI score, compared with only half of those treated with placebo and glucocorticoids. Patients treated with dupilumab also used less than half of the glucocorticoids used by patients treated with placebo.

For Beck, the surprising result was the decreased itching observed in dupilumab-treated patients. "Until this therapy came out, l'm not sure that we would have thought IL-4 and IL-13 were the major drivers of itch," she says. Although the molecular cause of the itch is still unclear, she thinks it is likely to be something downstream of these two cytokines.

Richard Locksley, a professor in the Infectious Diseases Department at the University of California, San Francisco (UCSF), has studied IL-4 and IL-13 extensively in mouse models of $\mathrm{T}_{\mathrm{H}} 2$-mediated diseases. Given the robustness of the mouse data, he's not surprised by the efficacy shown in these trials. "I'm more in the believer camp that we can actually learn things from animal models," he says. Data from mouse models have suggested that these two molecules are major drivers of $\mathrm{T}_{\mathrm{H}} 2$-mediated allergic responses, and the strength of these findings probably piqued interest in the development of antibodies targeting the IL-4 and IL-13 receptors.

Although he's encouraged by the results of the dupilumab trial, he notes that long-term safety data are still lacking. "So far, these things have been pretty safe, but again based on the animal studies we know there are a lot of physiological roles for these cytokines," he says. "There are no humans without IL-4 or IL-13, and that kind of tells you that it probably evolved to do something."

Interestingly, there was no attempt to select for patients with a $\mathrm{T}_{\mathrm{H}} 2$-enriched phenotype in the atopic dermatitis trial, whereas in the asthma trial only patients with elevated eosinophil levels (less than half of the patients assessed) were enrolled.

"Asthma may in fact be more heterogeneous than atopic dermatitis," explains Beck.

Particularly for asthma, "these medicines are going to be used in very specific, very well-selected groups of patients", agrees Fernando Martinez, director of the Arizona Respiratory Center. "One of the curses of developing new medicines in asthma is that we have very effective medicines now for probably $90 \%$ of patients," he says.

By contrast, there is currently no systemic treatment for atopic dermatitis. "Up to $50 \%$ of patients with atopic dermatitis no longer go to traditional health-care providers for their care because they have nothing new to offer," says Beck. Dupilumab, now in Phase III trials for asthma and atopic dermatitis, could be the news they've been waiting for. 